\title{
Energy Optimization for Large Scale Wireless Sensor Network using Real-Time Dynamics
}

\author{
M.C. Rajalakshmi \\ Research Scholar \\ University of Mysore, Mysore \\ Mysore, India
}

\author{
A.P Gnana Prakash \\ Associate Prof.: Dept. of Studies in Physics \\ Manasagangothri, University of Mysore \\ Mysore, India
}

\begin{abstract}
Energy depletion in Wireless Sensor Network (WSN) is one of the most focused research area in wireless network which is yet to witness a potentially significant mitigation techniques for ensuring substantial energy preservation. Owing to the resource constraints as well as low computational capability of the sensor mote, usually the existing energy conservation techniques finds its quite challenging to encapsulate variables of entire problem space. Hence, for the purpose of better mathematical formulation for energy efficiency solutions, it is necessary that all the real-time constraints should be empirically considered. Therefore, this paper presents a novel optimization technique that ensures sustainance of optimal network lifetime in large scale WSN considering the real-time dynamics. The outcome accomplished from the study is compared with standard and most frequently adopted energyefficient hierarchical routing algorithm to find that proposed system meets better criteria of energy preservation in large scale network.
\end{abstract}

\section{Keywords}

Wireless Sensor Network, Energy Issue, Energy Optimization

\section{INTRODUCTION}

The area of wireless sensor network has witnessed a tremendous revolution in the past decade due to its inherent capability of communication over the remote areas [1]. Wireless sensor network can be discussed under the special domain of communication engineering where the nodes are deployed in the regions which is not possible for performing human-based monitoring. The sensor nodes are basically an electronic device that has the capability of sensing a physical attributes like heat, moisture, motion, smoke etc. The wireless sensor network has also wide varieties of application ranging from ecological habitat monitoring to the defence application system. The study of WSN is always composed of three entities i) sensor nodes, and ii) cluster head, and iii) Base station [2]. Basically, the job of sensor node is to acquire the physical attributes which may be time based or frequency based. The cluster head is also a sensor node who collects the unique and non-redundant data and forward it the base station. This forwarding of the unique and non-redundant data from cluster head to base station is also called as data aggregation. However, it should be noted that the sensor mote are very small in nature with very limited computing capabilities. It also has limited storage facilities. Because of this constraints, there are various issues of WSN viz. routing issues, bandwidth issues, throughout issues, security issues etc. Out of the various issues that have been studied in the past, energy is one of the prominent and yet unsolved issues in the research communities. There were various formulations of design, algorithms, and mathematical model in the past that has totally focused on solving the energy issue in order to maximize the lifetime of the WSN. However, very few of the prior work have received recognition. Out of the majority of the work introduced in the past, LEACH [3] is considered as highly standard and benchmarked formulation till data. May be this is one of the reason, why $97 \%$ of the outcome of the existing research work focusing on energy issues is compared with LEACH algorithm.

Although there are various versions of LEACH algorithm, but major research communities select LEACH model as the standard work because of the design of the radio model and energy model. Various components that maps with the physical entities in the sensor mote is considered in the LEACH protocol. However, various studies also proved that LEACH is not the appropriate protocol to mitigate the energy issues. Hence, this fact gave rise to various other research work addressing the similar energy issues using Artificial Neural Network [4], Genetic Algorithm [5], Fuzzy Logic [6], Swarm Intelligence [7], game theory [8] and many other advanced technologies. However, till date none of the prior studies has managed to optimize the cumulative network lifetime of the WSN. This study therefore attempts to investigate the root cause of the energy depletion and introduces an empirical formulation that assist to optimize the cumulative network exponentially. The proposed study digs into the energy model preliminarily where the various constraints of the sensor motes were discussed with mathematical variables and optimization condition has been derived. Not only this, the model also considers a fact that if the cluster head is appropriately selected and ensures participation of other cluster heads too, exclusively in large scale wireless sensor network, than there is a fair possibilities of energy optimization in cost effective manner. Section-2 gives an overview of related work which identifies all the major research work being done in this area. Section 3 highlights problem formulation of the proposed study considering various real-time constraints and limitation of WSN. Section 4 discusses about the proposed model. Section 5 discusses about the implementation technique and result discussion. This section also discusses about the algorithm that has been used for the study along with various outcome of the study for the purpose of analyzing the effectiveness of the outcome. Finally, Section 6 summarizes the present paper.

\section{RELATED WORK}

Khan [9] presents a survey for designing energy efficient Wireless Sensor Network (WSN) Protocols and identifies future trends in designing energy efficient WSN protocols. Singh and Arora [10] demonstrated, the two major applications of the wireless sensor network are tracking based and second is monitoring based. There are sensors which communicate with each other to form a network. In the wireless sensor network there are number of issues which affects the performance and 
efficiency of the wireless sensor network. This paper covers almost all issues and simulation tools.

Gowrishankar et al. [11] presented an overview of ongoing research activities, various design issues involved and possible solutions incorporating these issues. This paper provides a cursory look at each and every topic in WSN and their main aim is to introduce a newbie to the field of WSN and make him understand the various topics of interest available for research. $\mathrm{Yu}$ et al. [12] proposed a layered architecture for each cluster that consists of a cluster control layer and a resource management layer. Key design issues and related challenges within this framework that deserve further investigation are outlined. Finally, they discuss a technique for energy-efficient resource allocation in a single-hop cluster, which serves as the basic primitive for the development of the resource management layer.

Ohize [13] focused on presenting to the reader emerging issues in wireless sensor networks. I have concentrated on grouping emerging issues in Wireless sensor network into three broad groups namely; the system group issues, the communication protocol issues and the service group issues.

Paschalidis et al. [14] formulated the combinatorial optimization problem of selecting such a minimal energy tree as a mixed integer linear programming problem. Since the problem is NP complete they devise a semi-definite relaxation and establish bounds on the optimal cost. They also develop a series of graph based algorithms that yield energy efficient bidirectional spanning trees and establish associated bounds on the optimal cost.

Mhatre et al. [15] presented a survey of some of the recent work on energy and cost optimizations in wireless sensor networks. Sensor nodes are characterized by severe energy budget due to limited battery life. They focus on two main problem areas, namely routing and design.

Yen et al. [16] propose a new controlled layer deployment (CLD) routing protocol to guarantee coverage and energy efficiency on a sensor network. CLD outperforms PEAS (Probing Environment and Adaptive Sleeping) and the TTDD (Two-Tier Data Dissemination) protocols in that it can guarantee full area coverage and connection. Finally, they show the results of the simulation to prove that the new protocol can use fewer sensor nodes for coverage and increase the lifetime as compared to the PEAS protocol.

Correia et al. [17] proposed a preliminary study on the use of QoS in WSN. They focus their research on QoS techniques applied to medium access control (MAC), and propose a classification of such techniques in four categories.

Diallo et al. [18] illustrated an effective manner of using the LQI which is among the best perform ant criteria. They also propose a simple mechanism to reduce single-node cluster phenomena which highly enhances the energy efficiency.

Anjali and Kaur [19] demonstrated propose a new approach to provide multiple routing paths using an Ant Colony Optimization (ACO) algorithm. Ant Colony Optimization based routing algorithms have been proposed to solve the routing problem trying to deal with these constrains. This paper will find an energy efficiency path in the WSNs using a method is based on Ant Colony Optimization (ACO) algorithms, aiming to minimize the consumption of power, improve fault tolerance, and length the network lifetime.

Sha et al. [20] presented an adaptive energy detection protocol for LPL, which dynamically adjusts a node's CCA threshold to improve network reliability and duty cycle based on application-specified bounds. Empirical experiments in both controlled tests and real-world environments showed AEDP can effectively mitigate the impact of noise on radio duty cycles, while maintaining satisfactory link reliability.

Faujadar [21] studied various energy-efficient routing algorithms, and compare among themselves. They take into account the setup costs and analyze the energy efficiency and the useful lifetime of the system. In order to better understand the characteristics of each algorithm and how well they really perform, they also compare them with an optimum clustering algorithm.

Sunita and Nandgave [22] summarized recent research results on data routing in sensor networks which comes under QOS based category They also included in the table whether the Protocol is utilizing flat or multihop transmission scheme, since it is an important consideration for routing protocols in terms of energy saving and traffic optimization.

Seah et al. [23] presented first review the recent developments in the area of Wireless Sensor Networks Powered by Ambient Energy Harvesting (WSN-HEAP). Then, they discuss the challenges faced by researchers in designing networking protocols for WSN-HEAP and summarize the open research problems.

Joze et al. [24] demonstrated that single-hop transmission is more efficient, when power consumption of real wireless sensor node's transceivers is taken into account. They also presented Node can route its messages towards destination either by using small or large hops.

Mathioudakis et al. [25] presented the key goal was to study the effect of MAC protocols for WSNs, in terms of energy consumption, throughput and energy efficiency. They have compared the performance of four widely used MAC protocols (IEEE 802.11, TDMA, SMAC and IEEE 802.15.4) used in sensor networks for energy conservation.

This section has presented couple of discussion of various authors about the technical solution towards mitigating the energy issues in WSN. However, very few of the solutions are found to be standardized or benchmarked. Hence, this paper has presented a cost effective technique that attempts to introduce an empirical model toward energy optimization designed for large scale WSN.

\section{PROBLEM FORMULATION}

For the purpose of evaluating the core issues associated with multi-level optimization for the purpose of enhancing the cumulative lifetime of the WSN, it is very critical to understand the empirical approach of data aggregation process and loopholes in unscheduled energy depletion. Therefore, this section will present the problem formulation of the study that will be aimed to solve for optimizing power conservation as an anticipated outcome of the study.

Table 1. Notation used in the study

\begin{tabular}{|l|l|}
\hline Notation & Meaning \\
\hline $\mathrm{N}_{\text {sen }}$ & Number of sensor motes \\
\hline $\mathrm{BS}$ & Base Station \\
\hline $\mathrm{R}_{\mathrm{i}}$ & Rate \\
\hline $\mathrm{P}_{\mathrm{BS}}$ & Position of base station \\
\hline $\mathrm{T}_{\mathrm{p}}$ & Temporary stay time \\
\hline
\end{tabular}


Consider a large scale wireless sensor network environment with specific number of nodes Nsen and BS as base station performing the data aggregation from the specific cluster heads residing the simulation environment. As the study considers larger scale of wireless sensor network, so the assumptions of random distribution of the sensor motes are considered for the problem formulations. It is considered that once the sensor nodes are randomly distributed in the simulation area, the sensors continuously keep on collecting their environmental sensing data keeping their initial position of the distribution constant. This fact will mean that nodes are static in their preliminary locus of distribution. Consider that the data is generated at a rate of $R_{i}$ (where $\left.R_{i}>0\right)$ for ith sensor mote. For understanding the challenges in the power conservation issues during data aggregation process, the study considers different position of the base station to find the optimality of the energy conservation. Hence, the study also considers the mobility of the base station. However, it should be known that possibility of base station position and sensor mote position may not be same at a given time. Consider PBS be the position of the base station and its temporary stay time be designated as $T p(p \in P)$. Therefore $T_{p}$ will mean the cumulative stay time of base station in position $\mathrm{p}$. The study doesn't consider the possibility of time required to travel by base station from one to another position as it may be significantly smaller. The system also considers the multiple hops which will be required by the motes in order to perform communication with the base station as exhibited in Fig.1

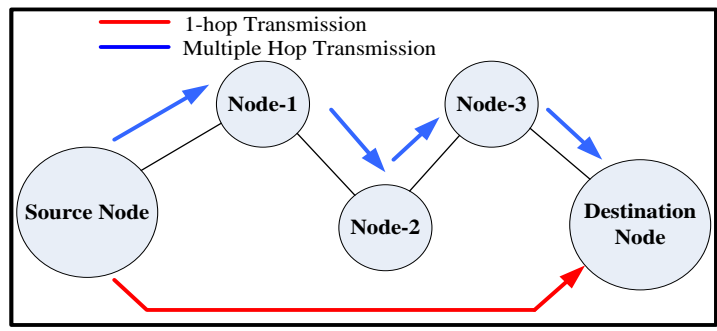

Fig 1: Multiple Hop Communication

This fact also means that with a given position of $\mathrm{p}(\mathrm{p} \varepsilon \mathrm{P})$, the base station is not always required to be within the transmission zone of other sensors. Consider a group of nodes $G_{i}^{p} \subseteq N_{\text {sen }} \cup\{g\}_{\text {that can }}$ be possibly a member sensor node or base station residing at the transmission zone of the sensor node $i$ ( $i \in$ Nsen) for a given position $p(p \in P)$ of the base station. Therefore, if $j\left(j \in G_{i}^{p}\right)$ than $j$ can be termed as neighbor node of $i$ for position $p$. It can be also seen that only the constituent that can differ among any two groups $G^{p 1}{ }_{i}, G^{p 2}{ }_{i}, p_{1}$, $\mathrm{p}_{2} \in \mathrm{P}$ is the base station $\mathrm{BS}$ as remaining of the network remains static.

The next phase of the problem formulation will be with respect to the energy variable as every sensor mote $i \in \mathrm{Nsen}$ is considered to posses certain initial energy $E_{i}>0$. The study also considers that the base station doesn't have any such constraint of the energy factor and is constantly receives the power supply. The energy model considers transmittance energy $\mathrm{E}_{\mathrm{TX}}>0$ that is drained by a sensor node $\mathrm{i}$ to forward a specific data to its neighborhood node (node within the transmission region). Similarly, the system consider $\mathrm{E}_{\mathrm{RX}}>0$ be the energy required to received the data packet from node $i$ to node $j$. The formulation of such energy model is also inspired by the work of Heinzelman [3] who is the pioneer of LEACH algorithm. However, for the easiness in the computation, the system doesn't consider any physical distance between any two nodes and other factors related to signal attenuation while performing data aggregation in WSN. Using the network and standard energy model, the system is about to formulate the environment of data aggregation so that energy behaviour can be studied and possible enhancement could be done using optimization principles. In doing so, there are two critical need that the topology should posses and they are i) provisioning operation to identify for every position $p(p \in P)$ in the timeinstant $\mathrm{Tp}$ for which the base station reside in that position, and ii) routing operation for investigating the cumulative estimated energy efficient path from sensor motes to the base station for every position $\mathrm{p}(\mathrm{p} \in \mathrm{P})$ for which $\mathrm{T}_{\mathrm{p}}>0$.

As the concept of problem formulation has mechanized mobility of the base station to alleviate the energy drainage issues during data aggregation, hence importance should be given to the position factor of the base station. Consider the rate of information transfer from sensor node $\mathrm{i}$ to neighbor node $\mathrm{j}$ be $\mathrm{R}_{\mathrm{i}}^{\mathrm{p}}$, to be allocated by the routing operation during the time duration $\mathrm{T}_{\mathrm{p}}$. For each $\mathrm{R}_{\mathrm{i}}^{\mathrm{p}}$, there exists a constraint $\mathrm{R}_{\mathrm{i}}^{\mathrm{p}}<$ $\mathrm{R}_{\max }$, where $\mathrm{R}_{\operatorname{maxi}}$ is the maximum feasible rate of information transfer from node $\mathrm{i}$ to node $\mathrm{j}$. Such hurdles can be visualized as constraints of route capacity that can be possibly investigated from the network environment. Along with consideration of such limitations associated with the strength of the links, there exists energy limitation too for every sensor. Hence, the study considers a strong link only between two nodes with maximum residual energy. It can also be said that for every position of the base station $p \in P$, the extent of the energy depletion at the source sensor $i \in$ Nsen is incurred by the transmittance and the receiving of node $i$ during the time instant $T_{p}$ that can never go beyond the maximum score of $E_{i}$. Interestingly, this value can exhibit the limitations / constraints imposed by the physical properties of the sensor node that are not considered in this simulation study.

\section{PROPOSED SYSTEM}

After visualizing the problem formulation of the study in the previous section, it can be said that cumulative focus of the study is to optimize the core duration for effectively performing the network operations before witnessing First Node Death (FND). It can be alternatively said that the study attempts to delay the instances of FND for large scale wireless sensor network. The proposed study makes the concrete definition of the network lifetime by equating it to sum of all the time required for temporary stay of the base station at all the feasible locations. The time of temporary stay is again limited by the another assumption of the study which states that the cumulative depleted energy by each of the sensor node, when the base station stays at various positions, should not go beyond the initial amount of energy in the sensor node. The study of the proposed system also considers the limitation factor of the energy of individual sensor mote and rate of transmission. Hence, the study considers designing of an empirical model that attempts to perform optimization of the energy in the standard energy model of WSN that directly intents to maximize the cumulative network lifetime of WSN.

Hence, the preliminary consideration of the proposed empirical model related to energy related attribute is that extent of energy depletion is directly proportional to the rate at which the data are either transmitted or received. Considering the stay time of the base station $T p$ and the data rate transfer $R^{p}{ }_{i}, i \in N_{\text {sen }}, j \in G^{p}$, $p \in P$, the energy depleted at the sensor mote $i$ when the base station is positioned at the locus $\mathrm{p}$ is thereby represented as,

$$
E=E_{T X}+E_{R X}
$$




$$
E=\sum_{j \in G_{i}^{p}} E_{T X} \cdot R_{i, j}^{p}+\sum_{j: i \in G_{j}^{p}} E_{R X} \cdot R_{j, i}^{p}
$$

It can be therefore seen that the above equation (1) has been formulated with the aid the cumulative receiving / transmitting energy with respect to the data transfer rate. However, if the time duration $\mathrm{Tp}$ is considered for the purpose of evaluating the energy depletion, than the equation (1) can be amended as below,

$$
E=\left(\sum_{j \in G_{i}^{p}} E_{T X} \cdot R_{i, j}^{p}+\sum_{j: i \in G_{j}^{p}} E_{R X} \cdot R_{j, i}^{p}\right) T_{p}
$$

Hence, the cumulative energy depleted at the mote i during the data aggregation can be represented by the sum of the all the individual elements in RHS of eq(2) with Tp over all the position $\mathrm{p} \epsilon \mathrm{P}$, i.e.

$$
E=\sum_{p \in P} \sum_{j \in G_{i}^{p}}\left(E_{T X} \cdot R_{i, j}^{p}\right) T_{p}+\sum_{p \in P} \sum_{j: i \in G_{j}^{p}}\left(E_{R X} \cdot R_{j, i}^{p}\right) T_{p}
$$

Using the principle instances of FND, the cumulative energy factor of the large scale WSN environment can be represented as the dimension of time instance till occurrence of FND. It can also be represented as the summation of the temporary stay time of the base station at all the feasible position. Hence, this fact leads to next target of the study which is to identify the temporary stay time of the base station $\mathrm{Tp}$ and the rate of data transfer Rpi that could possibly increase the lifetime of the network under the flow conservation condition and considering the limitation that cumulative energy drained by the sensor mote when the base station resides in multiple position cannot go beyond the initial energy of the sensor node. Hence, with the support of such empirical evidences, the problem formulation of the proposed system can be designated as the problem of optimizing the network lifetime of large scale sensor network.

$$
\text { Maximize } \sum_{p \in P} T_{p} \text { subjected to } T_{p} \geq 0, p \in P
$$

$$
\begin{gathered}
0 \leq R_{i j}^{p} \leq R_{i j}^{\max }, i \in N_{s e n}, j \in G_{i}^{p}, p \in P \\
\sum_{p \in P} \sum_{j \in G_{i}^{p}}\left(E_{T X} \cdot R_{i, j}^{p}\right) \cdot T_{p}+\sum_{p \in P} \sum_{j: i \in G_{j}^{p}}\left(E_{R X} \cdot R_{j, i}^{p}\right) T_{p} \leq E_{i} \\
\sum_{j: i \in G_{j}^{p}} R_{j i}^{p}+R_{i}=\sum_{j \in G_{i}^{p}} R_{i j}^{p}, i \in N_{s e n}, p \in P
\end{gathered}
$$

It can be seen that the condition for the flow conservation exhibited in eq (7) is applicable to all the possible position of the base station $\mathrm{p} \in \mathrm{P}$ individually. This fact will eventually mean that for all the position of the base station, the cumulative inbound rate of data packets transmission added to the rate of the data packet generation at a sensor mote is equivalent to the cumulative outbound rate of the data packet transmission from the individual sensor mote. As the base station is the only terminal where the aggregated data is accumulated, so following empirical facts will hold true,

$$
\begin{aligned}
& R_{B S, j}^{p}=0, j \in N_{\text {sen }}, p \in P \\
& R_{B S}=-\sum_{i \in N_{\text {sen }}} R_{i}
\end{aligned}
$$

Hence, by highlighting $\overline{R_{i j}^{p}}=R_{i j}^{p} \cdot T_{p}$ as the quantum of the data being transmitted from node $i$ to node $j$ during time $T_{p}$, the problem pertaining to optimization of energy can be now represented as,

$$
\begin{gathered}
\text { Maximize } \sum_{p \in P} T_{p} \text { subjected to } T_{p} \geq 0, p \in P \\
0 \leq \bar{R}_{i j}^{p} \leq R_{i j}^{\max } \cdot T_{p}, i \in N_{s e n}, j \in G_{i}^{p}, p \in P \\
\sum_{p \in P} \sum_{j \in G_{i}^{p}} E_{T X} \bar{R}_{i, j}^{p}+\sum_{p \in P} \sum_{j: i \in G_{j}^{p}} E_{R X} \bar{R}_{j, i}^{p} \leq E_{i} T_{p_{i}} \\
\sum_{p \in P} \sum_{j \in G_{i}^{p}} E_{T X} \cdot \bar{R}_{i, j}^{p}+\sum_{p \in P} \sum_{j: i \in G_{j}^{p}} E_{R X} \cdot \bar{R}_{j, i}^{p} \leq E_{i} \\
\sum_{j i: G_{j}^{p}} \bar{R}_{j i}^{p}+R_{i} \cdot T_{p}=\sum_{j \in G_{i}^{p}} \bar{R}_{i j}^{p}, i \in N_{s e n}, p \in P
\end{gathered}
$$

Hence, using optimization principles, it can be said that using objective (9), the cumulative network lifetime can be enhanced in large scale wireless sensor network that has considered the summation of temporary stay time of base station at all the feasible positions, while the empirics $T_{p}>0$ ensures the positive value of $\mathrm{Tp}$ at $\mathrm{p} \in \mathrm{P}$. By evaluating the product of transmission rate constraint eq. (5) with the energy of the sensor nodes eq. (6) by the variable $\mathrm{Tp}$, the outcome of (10) and (11) is accomplished respectively. However in the equation (12), the inequality in LHS exhibits the cumulative amount of the energy depleted from node $\mathrm{i}$ to transmit the data packet to node $\mathrm{j}$ considering all the temporary stay time of the base station at the position visited. Therefore, equation (12) highlights the energy limitations of the sensor motes that should not go beyond the initial energy of the $E_{i}$ of that particular sensor Thereby by multiplying equation (7) with $\mathrm{Tp}$, the outcome can be observed in equation (13)

Therefore, it can be seen that above optimization model is capable of evaluating all the possible scenarios of mobility of the base station considered in large scale WSN at duration $T_{p}$ for which the base station reside at that position of the simulation environment and thereby the cumulative network lifetime can be optimized. Using the model, it is feasible for evaluate the rate of data transmission as

$$
R_{i j}^{p}=\frac{\bar{R}_{i j}^{p}}{T_{p}}
$$

and based on the above equation (14), it can be said that proposed model will provide the optimization to both provisioning as well as routing. Considering the worst scenario of mobility where the possibility of energy drainage is much, the model is therefore capable of higher degree of energy conservation in large scale network. In this scenario, if the maximum value of the Tp is zero, the possibility of base station to visit the position $\mathrm{P}$ is almost void. 


\subsection{Selection of Cluster Head}

The design of the decision rule using probabilistic approach for selection of cluster head is basically aimed to provide energy efficient data aggregation process. For this purpose, the proposed study considers two conditions for selection of cluster head as discussed below:

\subsubsection{Condition-1}

This condition is one of the most significant in the design of the connected probabilistic parameters, where the probabilistic sets evaluates the multiple information of the cluster head with respect to distance among the member nodes and their residual energy. The node with highest residual energy is considered as cluster head in the preliminary rounds. Any nodes with less residual energy are rejected for the selection process.

\subsubsection{Condition-2}

The assumptions of the previous condition may not be purely wise enough as it may not be applicable to different topologies as well as applications of the WSN. Hence for better extensibility, the study also chooses to formulate an alternative strategy beyond the energy attribute for choosing the cluster head more wisely. The formulation of this condition is to ensure that all the cluster head do participate in data aggregation process. As the study is formulated for the large scale WSN where the possibility of the cluster head is enormously high with the increasing number of the cluster. Moreover, as the study considers random distribution of the sensor nodes, it is quite possible that some cluster head may not actively participate in the data aggregation process for which reason the cumulative network lifetime may be reduced. This condition of the design of the probabilistic approach connected parameters considers an optimal position of the sensor motes which is now considered as cluster head from the previous stage. The secondary priority is focused on unique distribution of data packets at the instant of congested network condition. The base station chooses every wireless sensor node and estimates the sum of the squared distances of other cluster head from those selected nodes. As the energy required for transmission is directly proportional to $\mathrm{d} 2$, hence it lowers the amount of energy needed by other cluster heads for forwarding the aggregated data through that cluster head. The formulation of the second condition also permits the checking traffic to increase the gross network lifetime and in order to accomplish this fact, a relative distance estimation of cluster head to another node (may be cluster head or base station) is estimated.

\section{IMPLEMENTATION AND RESULT DISCUSSION}

The basis of the proposed study is the essentials of the empirical model that is described in the previous section, hence, due to inclusion of mathematical theories, the study was decided to be carried using Matlab as a base of programming as it has some rich functions and methods which will minimize the coding complexities. The experimentation was carried out in Windows operating system on 32 bit machine using core i5 processor. Although, it is expected that it must substantially work in the prior versions, but due to extent of computation, the study considers the latest versions of computing resources. The proposed work is experiment on a simulation area of 1200 x $1500 \mathrm{~m} 2$. The studies have also considered a numerous scenarios with 500-1000 nodes with random distribution. The preliminary energy of cluster head is considered as 0.5 Joules with 200 rounds of iteration. The proposed system is compared to frequently used LEACH [3] protocol. The network simulation parameters considered are as shown below:
Table 1. Simulation Parameters

\begin{tabular}{|l|l|}
\hline Total Number of Nodes & $500-1000$ \\
\hline Size of network & $1200 \times 1500 \mathrm{~m}^{2}$ \\
\hline Preliminary Energy Initialization & $0.5 \mathrm{~J}$ \\
\hline Size of data-packet & $5000 \mathrm{bits}$ \\
\hline
\end{tabular}

This is one of the significant sections of the comparative analysis that performs checking of the efficiency of the proposed empirical scheme that is design for the proposed system. The deployment of the schema should maximize the throughout along with minimization of the unwanted energy depletion issues in large scale WSN, which is the significant concern of this research work. The parts of the rule are designed in such a way that second condition is highly enhanced version of the first condition using probabilistic approach. The algorithm that is responsible for performing energy optimization in the proposed study is discussed as below:

Algorithm: Energy Optimization in WSN

Input:

Output:

Step:

1. Initialize the simulation parameters

2. Design the Energy Model

2.1 Initialize individual Energy Variable $\left(\mathrm{E}_{\mathrm{TX}}, \mathrm{E}_{\mathrm{RX}}\right)$ Estimate $\mathrm{E}=\mathrm{E}_{\mathrm{TX}}+\mathrm{E}_{\mathrm{RX}}$

2.2 Considering rate,

$$
E=\sum_{p \in P} \sum_{j \in G_{i}^{p}}\left(E_{T X} \cdot R_{i, j}^{p}\right) \cdot T_{p}+\sum_{p \in P} \sum_{j: i \in G_{j}^{p}}\left(E_{R X} \cdot R_{j, i}^{p}\right) T_{p}
$$

2.3. Maximize $\sum_{p \in P} T_{p}$

$$
\text { Subjected to } T_{p} \geq 0, p \in P
$$

2.3 For $i \in N_{\text {sen }}, j \in G_{i}^{p}, p \in P$

$$
\text { Estimate } \bar{R}_{i j}^{p}, 0 \leq \bar{R}_{i j}^{p} \leq R_{i j}^{\max } \cdot T_{p}
$$

2.4 Update, energy consumption w.r.t mobility

$$
\sum_{p \in P} \sum_{j \in G_{i}^{p}} E_{T X} \cdot \bar{R}_{i, j}^{p}+\sum_{p \in P} \sum_{j: i \in G_{j}^{p}} E_{R X} \cdot \bar{R}_{j, i}^{p} \leq E_{i} \cdot T_{p_{i}}
$$

2.5 Evaluate the rate of transmission w.r.t $\mathrm{T}_{\mathrm{P}}$

$$
\sum_{j: i \in G_{j}^{p}} \bar{R}_{j i}^{p}+R_{i} \cdot T_{p}=\sum_{j \in G_{i}^{p}} \bar{R}_{i j}^{p}
$$

26. Evaluate the rate of data transmission as

$$
R_{i j}^{p}=\frac{\bar{R}_{i j}^{p}}{T_{p}}
$$




\section{Design the $\mathrm{CH}$ selection Model}

\subsection{Estimate Residual Energy $=\sum \mathrm{E}_{\mathrm{i}} / /$ Cond -1}

\subsection{If $\mathrm{E}_{\mathrm{i}}<=0$, node dies}

\subsection{Estimate first node death;}

\subsection{Cluster_Head ALIVE $=\mathrm{N}_{\text {sens }}-\sum$ Cluster_Head}

3.5 Increment the rounds of data transmission.

3.6 Estimate sum of squared distances of other $\mathrm{CH}$

3.7 Lower down $\mathrm{E}_{\mathrm{TX}}$ and $\mathrm{E}_{\mathrm{RX}}$

\subsection{Update new energy $\mathrm{E}_{\mathrm{i}}$}

\section{End}

By applying the above algorithm, the proposed model assists not only in selection of efficient cluster head, but it also assists in enhancing the cumulative lifetime of the network, especially when the study is totally focused on large scale wireless sensor network. While comparing with the conventional LEACH algorithm, the proposed system is checked for 3 different performance evaluation parameter e.g. i) Energy Retention, ii) Rate of Energy Optimized, and iii) Throughput. Figure 2 exhibits the extent of energy retention for the LEACH as well as proposed system. Energy retention is estimated from the cumulative residual energy considering the $T_{P}$ and various parameters in the simulation. The fig. 2 shows that instant of energy depletion is witnessed around 400 iteration which is almost the better version of LEACH. Moreover due to the formulation of the efficient strategies of $\mathrm{CH}$, this doesn't only consider energy but also consider various other parameters. Due to the assurance of inclusion of large number of $\mathrm{CH}$ in data aggregation process, the proposed system was obviously witnessed with higher residual energy, where the descends of the curve is very much gradual. Hence, better energy retention is observed.

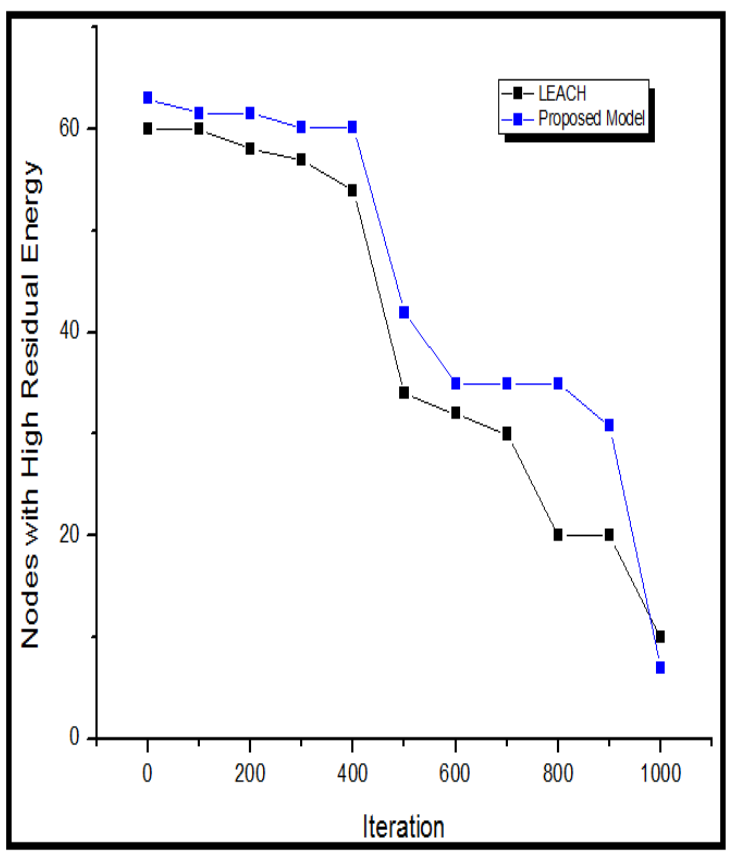

Fig 2: Performance Evaluation of Energy Retention

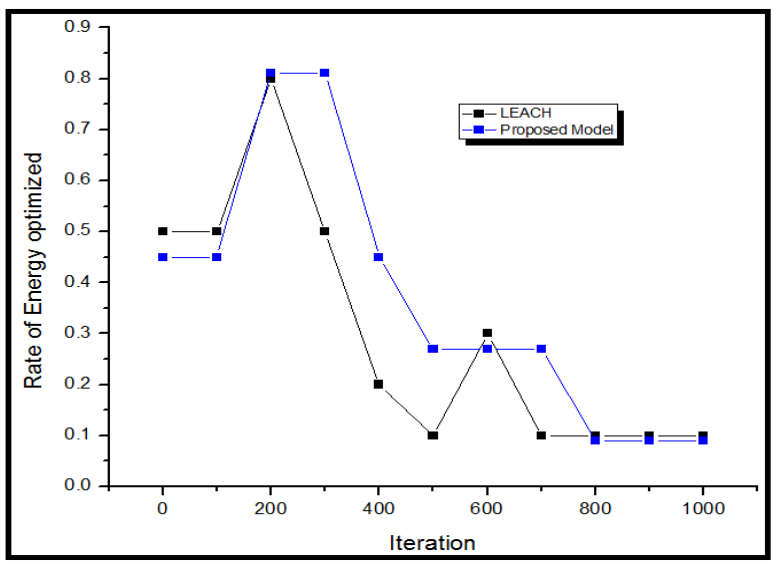

Fig 3: Performance Evaluation of the Rate of Energy Optimized

Figure 3 represents the performance evaluation analysis for the rate of energy optimized. Basically, the rate of energy optimized is evaluated by calculating the total number of alive nodes and its occurrence of FND. Hence, y-axis for Fig.3 represents the energy in joules which is estimated in due progress of simulation rounds of 1000 . The result also shows that there is a huge fluctuation of the LEACH algorithm where the variance of energy factor is found initially higher and then it has a gradual degradation, whereas in the proposed system, the initial performance of the energy optimization was very less compared to LEACH, but with the increase of the number of the iteration, it is found with much better outcome as compared to LEACH. This outcome can eventually interpret that outcome of LEACH is outnumbered by proposed model which has witnessed higher rate of energy optimization. Indirectly, it also says that proposed model has successfully delayed the instance of FND.

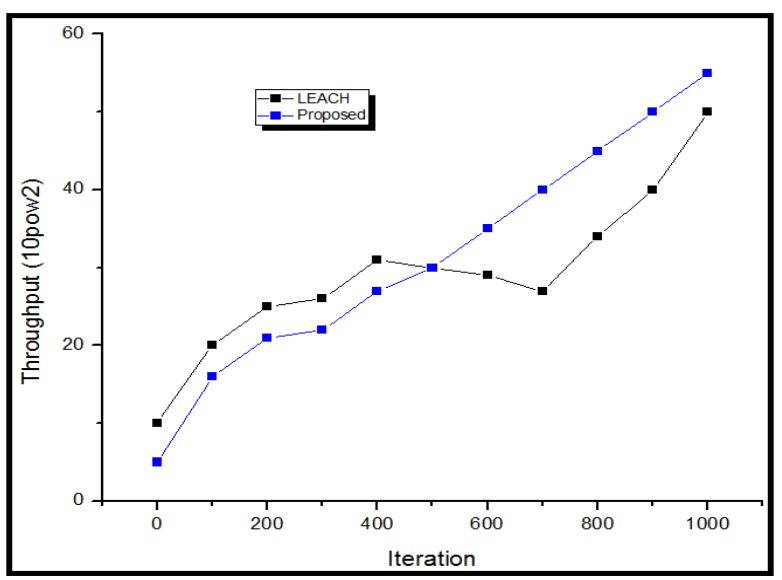

Fig 4: Performance Evaluation of the Throughput

Figure 4 exhibits the performance evaluation of the throughout. Basically the term throughput pertains to the packet delivery ratio in terms of bytes. The outcome shows that proposed empirical formulation of the proposed system has never been an impediment towards increasing the throughput of the system. Another basic reason for the enhanced throughput of the proposed model is that the system has fair use of rate of transmission of data packet which has been duly optimized along with the energy optimization. Occurrence of such phenomenon is lacking in LEACH protocol and therefore after 400 iterations, it is found that outcome of LEACH protocol is outnumbered by the proposed model. Hence, the proposed model truly supports large scale wireless sensor network environment. 


\section{CONCLUSION}

Modelling the solution for energy efficiency in WSN is one of the challenging task, especially when it is considered for large scale network. As in large scale network, there are various realtime factors that significant affects the energy stability among a large number of sensor nodes considering multi-hop communication system. The proposed study introduces a mathematical modelling of energy efficiency technique considering this aspect by i) formulating empirical representation of energy depleted at sensor mote during data aggregation, ii) framing up condition for optimizing the network lifetime for large scale WSN, and iii) rate of data transmission. The proposed technique also discusses about the conditions required to select cluster head to ensure that energy is conserved maximally during and after selection of cluster head. The simulation being conducted with 500-1000 sensor motes shows that proposed system have optimal energy retention, better energy optimization rate, and higher throughput as compared to the most frequently adopted energy efficient hierarchical routing protocol.

\section{REFERENCES}

[1] Singh, G., Arora, H.2013. Design and Architectural Issues in Wireless Sensor Networks. International Journal of Advanced Research in Computer Science and Software Engineering, Vol.3, Issue. 1

[2] Josephine, J., Jebarajan, T., Rajesh, R.S.2013. An Energy Aware Key Establishment Framework for Wireless Sensor Network Security. International Journal of Scientific and Research Publications.Vol. 3, Issue. 2

[3] Heinzelman, W.R., Chandrakasan, A and Balakrishnan, H.2000. Energy-Efficient Communication Protocol for Wireless Microsensor Networks. Proceedings of the Hawaii International Conference on System Sciences

[4] Hosseingholizadeh, A.2009. A Neural Network approach for Wireless sensor network power management. Proceedings of 2nd International Workshop on Dependable Network Computing and Mobile Systems, Niagara Falls, USA

[5] Liu, J-L and Ravishankar, C.V.2011. LEACH-GA: Genetic Algorithm-Based Energy-Efficient Adaptive Clustering Protocol for Wireless Sensor Networks. International Journal of Machine Learning and Computing. Vol.1, No. 1

[6] Haider, T., Yusuf, M.2009. A Fuzzy Approach to Energy Optimized Routing for Wireless Sensor Networks. The International Arab Journal of Information Technology, Vol. 6, No. 2

[7] Iram, R., Sheikh, M.I., Jabbar, S., Minhas, A. A.2011. Computational Intelligence Based Optimization of Energy Aware Routing in WSN. Proceedings of the World Congress on Engineering and Computer Science.Vol. I

[8] Valli, R., Dananjayan, P. 2010. A non-cooperative game theoretical approach for power control in virtual mimo wireless sensor network. International Journal Of UbiComp (IJU), Vol.1, No.3

[9] Khan, A.R.2010. Energy Efficient Protocol Design Issues in Wireless Sensor Networks. Journal of Information \& Communication Technology, Vol. 4, No. 1

[10] Singh, G., Arora, H.2013. Design and Architectural Issues in Wireless Sensor Networks. International Journal of
Advanced Research in Computer Science and Software Engineering. Vol. 3, Issue. 1

[11] Gowrishankar, S., Basavaraju, T.G., Manjaiah, D.H., Sarkar, S.K.2008. Issues in Wireless Sensor Networks. Proceedings of the World Congress on Engineering, Vol I

[12] Yu, Y., Krishnamachari, B., Prasanna, V.K.2004. Issues in designing middleware for wireless sensor networks. IEEE Network, Vol. 18 (1)

[13] Ohize, H.O., Ohiani, H.2011. Emerging Issues in Wireless Sensor Networks. Journal of Software \& Automation

[14] Paschalidis, I.C., Binbin, L.2011. Energy optimized topologies for distributed averaging in wireless sensor networks. IEEE Transactions Automatic Control

[15] Mhatre, V., Rosenberg, C.2005. Energy and cost optimizations in wireless sensor networks: A survey. Performance Evaluation and Planning Methods for the Next Generation Internet, Springer US, pp. 227-248

[16] Yen, Y-S., Hong, S., Chang, R-S., Chao, H-C.2007. An energy efficient and coverage guaranteed wireless sensor network. IEEE Wireless Communications and Networking Conference

[17] Correia, L.H.A., Macedo, D.F., Santos, A.L.D.2005. Issues on QoS Schemes in Wireless Sensor Networks. DCC/UFMG, Tech. Report: RT 700.004

[18] Diallo, C., Marot, M., Becker, M.2010. Single-node cluster reduction in wsn and energy-efficiency during cluster formation. AdHoc Networking Workshop (MedHoc-Net), The 9th IFIP Annual Mediterranean.

[19] Anjali, N. Kaur.2013.A Review: Optimization of Energy in Wireless Sensor Networks. International Journal of Engineering Trends and Technology.Vol.4, Issue. 3

[20] Sha, M., Hackmann, G., Lu, C.2013. Energy-efficient low power listening for wireless sensor networks in noisy environments. Proceedings of the 12th international conference on Information processing in sensor networks

[21] Faujadar, P.S. (Retrrived Nov 2014).Synopsis of Energy Efficient Routing Protocols in Wireless Sensor Networks (WSN). Diss Indian Institute of Technology, Retreived from

cse.iitkgp.ac.in/ abhij/../Synopsis/03CS3006_Pawan_Sin gh_Faujadar.pdf

[22] Nandgave, S.S.2012. A Survey on QOS and energy efficient routing protocols in WSN. International Journal of Application or Innovation in Engineering \& Management. Vol. 1, Issue. 2

[23] Seah, W.KG., Arvin, T.S.2009. Challenges in Protocol Design for Wireless Sensor Networks Powered by Ambient Energy Harvesting. IEEE Wireless Communication

[24] Pesovic, U.M., Mohorko, J.J., Benkic, K. , Cucej, Z.F.2010. Single-hop vs. Multi-hop-Energy efficiency analysis in wireless sensor networks. 18th Telecommunications Forum, TELFOR

[25] Mathioudakis, I., White, N.M., Harris, N.R., Merret, G.V.2008. Wireless sensor networks: A case study for energy efficient environmental monitoring. Eurosensors, Dresden, Germany 